\title{
Schlafapnoescreening - reicht eine Messung?
}

\author{
Screening Measurements for Sleep Apnea: Is a Single Measurement Enough?
}

\section{Zusammenfassung}

Bei Patienten mit Schlafapnoesyndrom wurde bei Messwiederholungen teilweise eine hohe Variabilität der respiratorischen Atemstörung festgestellt. In der folgenden Studie wurde prospektiv die Variabilität des Apnoe-/Hypopnoeindex (AHI) unter ambulanten Bedingungen bestimmt. Dabei nahmen die Patienten nach einmaliger Anleitung selbständig Messungen über drei Nächte vor. Weiterhin wurde der Einfluss der Variabilität in bezug auf die erste Nacht (first night effect $=$ FNE), die Lageabhängigkeit (body position), den Schweregrad des SAS sowie das Alter und das Geschlecht der Patienten untersucht. Bei 19 Patienten (12 Männer und 7 Frauen, Alter 51,6 \pm 12,4 Jahren) mit anamnestischem Verdacht auf ein obstruktives Schlafapnoesyndrom wurde eine ambulante Messung (Jaeger Pro, Fa. ViaSys, Würzburg) an 3 aufeinanderfolgenden Nächten durchgeführt. Bezüglich des Apnoe-/Hypopnoeindex (AHI) und der kardiorespiratorischen Parameter unterschieden sich die Ergebnisse über die 3 Nächte statistisch nicht signifikant (AHI 1. Nacht 9,8 $\pm 11,7 / h, 2$. Nacht $10,1 \pm 9,6 / h, 3$. Nacht $8,5 \pm 9,0 / h$ ). Die individuelle AHI-Differenz betrug jedoch 5,6 $\pm 5,0 /$ h. Bei $15 \%$ der Patienten variierte der AHI sogar um mehr als 10 Ereignisse pro Stunde. Die Variabilität des AHI war mit dem Schweregrad der Befunde assoziiert. Ein FNE, eine Lageabhängigkeit der nächtlichen Variabilität des AHI sowie eine Assoziation mit dem Alter und Geschlecht der Patienten war nicht nachzuweisen. Bedingt durch die individuelle Variabilität des AHI wird bei Durchführung lediglich einer Messung bei einem erheblichen Teil der Patienten mit Schlafapnoesyndrom die Ausprägung des Befundes unterschätzt. Im klinischen Alltag kann durch Messwiederholung eine Optimierung der Aussagesicherheit erreicht werden.

\section{Abstract}

In patients with sleep apnea syndrome a high night by night variability of the respiratory breathing disturbance was reported in some studies. In the following study the variability of the apnoe-/hypopnoeindex (AHI) was prospectively determined with an ambulant monitoring system. Outpatients were instructed to perform three measurement at home independently. The influence of the variability was determined with respect to a first night effect (FNE), body position, severity of SAS, age and sex of the patients. 19 patients ( 12 men and 7 women, mean age $51.6 \pm 12.4$ years) were investigated. The measurements were done with the ambulatory device Jaeger pro (ViaSys Comp., Wuerzburg). No statistically significant differences of the AHI and the cardiorespiratory parameters between the 3 nights were found (AHI $9.8 \pm 11.7 / h, 10.1 \pm 9.6 / h$ vs. $8.5 \pm 9.0 / h$ ). However the individual AHI difference was $5,6 \pm 5,0 / \mathrm{h}$. In $15 \%$ of the patients the AHI varied even by more than 10 per h. The variability of the AHI was associated with the severity. No FNE, dependency of body position, sex or age was found. Due to the individual variability of the AHI severity in patients with sleep apnoe syndrome, the severity is underestimated in a considerable number of patients. Repetitive measurements improve the diagnostic power. 
In retrospektiven Untersuchungen konnte das Auftreten einer z.T. erheblichen individuellen Variabilität des Apnoe-/Hypopnoeindex (AHI) bei wiederholten nächtlichen Polysomnographien an Patienten unterschiedlichen Alters mit und ohne Symptome des Schlafapnoesyndroms gezeigt werden [1 -5]. Es wurden eine Reihe von Faktoren beschrieben, die die Variabilität bedingen können: Immer wieder wurde ein first night effect (FNE) nachgewiesen, bedingt durch ungewohnte oder veränderte Umgebungsbedingungen [6,7]. Andere Faktoren, die die Variabilität des AHI beeinflussen können sind Schweregrad der Erkrankung, die Abhängigkeit von der Lage, Alter und Geschlecht der Patienten $[8-11]$.

Zur Frage, mit welcher Ausprägung die Variabilität des AHI unter ambulanten Bedingungen auftritt, bei der die Patienten erstmals mit einer Messapparatur konfrontiert werden, wurde die folgende prospektive Studie durchgeführt, bei der Patienten mit klinischem Verdacht auf ein Schlafapnoesyndrom rekrutiert wurden, die die Messungen über den gesamten Messzeitraum selbständig ambulant durchführen.

\section{Patienten und Methoden}

Es wurden prospektiv und konsekutiv 30 ambulante Patienten, die sich wegen Verdacht auf ein Schlafapnoesyndom vorstellten, gemessen. Einschlusskriterien waren Tagesmüdigkeit mit einem Wert in der Epworth Sleepiness Scale größer 10 und Symptome von beobachtetem regelmäßigen Schnarchen und oder Atempausen, die in einer standardisierten Befragung erfasst wurden. Ausschlusskriterien waren Patienten mit COPD ( $\mathrm{FEV}_{1}<70 \%$ Soll), Herzinsuffizienz (Echokardiographisch FS < 30\%), Systemerkrankungen, maligne Tumorerkrankungen und neurologischen Erkrankungen. Das durchschnittliche Alter betrug 53,6 \pm 14,7 Jahren (21 Männer und 9 Frauen, BMI 28,4 $\pm 3,2 \mathrm{~kg} / \mathrm{qm}$ ). Zur weiteren Auswertung wurden nur Patienten aufgenommen, bei denen eine vollständige Messung über 3 Nächte gelang. Dies war bei 19 Patienten (12 Männer und 7 Frauen, Alter 51,6 \pm 12,4 Jahren, BMI $29 \pm 3,7 \mathrm{~kg} / \mathrm{qm})$ der Fall.

\section{Ambulantes Monitoring}

Mit dem Screeninggerät (Apnoescreen Pro, Fa Viasys, Würzburg) wurden Atemflow mit einem oronasalen Thermistor, thorakoabdominale Atembewegungen, Herzfrequenz, Sauerstoffsättigung und Körperlage aufgezeichnet. Die Aufnahmezeit des Rekorders betrug 3 Nächte.

Nachdem die Patienten der Durchführung der Untersuchung zugestimmt hatten, erhielten sie eine ausführliche Einführung, bei der sie mit der Handhabung des Gerätes vertraut gemacht wurden und das Anlegen der Sensoren erlernten. Sie wurden auf die Notwendigkeit hingewiesen, auf Alkohol sowie Sedativa zu verzichten. Anschließend nahmen sie das Gerät mit nach Hause und führten Messungen an 3 aufeinanderfolgenden Nächten selbständig durch. Während der Zeit bestand die Möglichkeit zu direktem Telefonkontakt.
Nach der Rückgabe des Gerätes erfolgte die Auswertung, die anhand der Ergebnisse der Computeranalyse sowie einer manuellen Auswertung am Monitor jeweils von derselben Person durchgeführt wurde.

\section{Auswertung der Ergebnisse}

Obstruktive Apnoen werden durch fehlenden oronasalen Luftstrom bei erhaltenen Thorax- bzw. Abdomenexkursionen definiert. Hypopnoen wurden durch eine Reduktion des oronasalen Luftstroms um mindestens $50 \%$ bei erhaltenen Thorax- bzw. Abdomenexkursionen und einen Abfall der Sauerstoffsättigung um mindestens $4 \%$ definiert.

Der maximale, minimale und durchschnittliche AHI jedes Patienten über die 3 Nächte wurde ermittelt und die maximale und durchschnittliche Länge der Apnoen bzw. Hypopnoen erfasst.

Die AHI-Differenz wurde bestimmt als Differenz zwischen maximalem und minimalem AHI. Der Variationskoeffizient des AHI wurde berechnet als Quotient aus dem durchschnittlichen AHI und der Standardabweichung. Ermittelt wurde die Anzahl der Entsättigungen und die Anzahl der Entsättigungen $<90 \%$ sowie der minimale $\mathrm{SaO}_{2}$-Wert und der durchschnittliche minimale $\mathrm{SaO}_{2}$-Wert. Außerdem wurde die Anzahl der Schnarchereignisse bestimmt sowie die Summe aus Tachykardie- (Herzfrequenz $>100 /$ min und länger als $10 \mathrm{~s}$ ) und Bradykardieereignissen (Herzfrequenz <60/min, > $10 \mathrm{~s}$ ) berechnet. Die Herzfrequenz wurde pulsoxymetrisch gemessen, Schlafzeiten und Schlafeffizienz wurden aktigraphisch abgeschätzt. Der AHI wurde auf die abgeschätzte Schlafzeit bezogen. Aufwacher wurden detektiert, wenn mehr als 50\% des maximalen aktigraphischen Ausschlags gemessen wurde.

Die durchschnittlichen Ergebnisse des AHI in 3 Messungen in der Gesamtgruppe wurden verglichen. Anschließend wurde die Variabilität des $\mathrm{AHI}$ in 2 Gruppen mit einem maximalen $\mathrm{AHI}<10 / \mathrm{h}$ bzw. $\geq 10 / h$ einander gegenübergestellt.

Die Lageabhängigkeit des AHI bedeutet, dass in Rückenlage ein mindestens 2 fach höherer AHI nachgewiesen wird als in Seitenlage. Um einen Zusammenhang zwischen der Lageabhängigkeit des AHI und der Variabilität des AHI zu untersuchen, wurde ein Vergleich der AHI-Differenz zwischen der Patientengruppe mit und ohne lageabhängigem AHI durchgeführt.

Die Variabilität des AHI wurde in 2 Altersgruppen mit einem Alter $<50$ Jahren bzw. $\geq 50$ Jahren sowie in 2 Patientengruppen mit männlichem bzw. weiblichem Geschlecht untersucht.

Es wurde die Sensitivität der Einzelmessungen sowie die Sensitivität jeweils zweier sowie aller 3 Messungen bestimmt.

\section{Statistische Analyse}

Statistische Tests wurden mit dem Programm SPSS (Version 10.1) durchgeführt. Die untersuchten Parameter erwiesen sich als nicht normalverteilt. Angesichts einer ausreichenden Robustheit des Testverfahrens wurde der nichtparametrische Test KruskalWallis zum Vergleich der Ergebnisse der 3 Messungen durchgeführt. 
Tab. 1 Ergebnisse der untersuchten Parameter in der Gesamtgruppe in den drei Messnächten

\begin{tabular}{|c|c|c|c|c|}
\hline$(n=19)$ & N1 & $N 2$ & N3 & $p$ \\
\hline$A H I(/ h)$ & $9,8 \pm 11,7$ & $10,1 \pm 9,6$ & $8,5 \pm 9,0$ & n.s. \\
\hline durchschn. Apnoe-Dauer (s) & $18,4 \pm 7,9$ & $17,9 \pm 4,6$ & $17,7 \pm 5,3$ & n.s. \\
\hline max. Apnoe-Dauer (s) & $39,3 \pm 24$ & $46,3 \pm 23$ & $43,4 \pm 29,1$ & n.s. \\
\hline durchschn. Hypopnoe-Dauer (s) & $30,4 \pm 10,3$ & $32,8 \pm 13,6$ & $28,4 \pm 15,8$ & n.s. \\
\hline max. Hypopnoe-Dauer (s) & $77,7 \pm 40$ & $77,2 \pm 38$ & $64,5 \pm 50,5$ & n.s. \\
\hline Entsättigungen (n) & $77,3 \pm 111,1$ & $69 \pm 83,1$ & $78,4 \pm 112,9$ & n.s. \\
\hline Entsättigungen < $90 \%(n)$ & $47,3 \pm 108,5$ & $41,4 \pm 75,7$ & $39,7 \pm 98,3$ & n.s. \\
\hline durchschn. minimaler $\mathrm{O}_{2}(\%)$ & $90,3 \pm 2,7$ & $90,3 \pm 2,2$ & $90,9 \pm 2,2$ & n.s. \\
\hline minimaler $\mathrm{O}_{2}(\%)$ & $82,4 \pm 9,3$ & $82,5 \pm 7,8$ & $85,1 \pm 7,5$ & n.s. \\
\hline Tachy-/Bradykardien (n) & $133,1 \pm 116$ & $101,6 \pm 84,7$ & $108 \pm 108,4$ & n.s. \\
\hline Schnarchereignisse (n) & $117,5 \pm 115,3$ & $108,6 \pm 106$ & $135,5 \pm 124,8$ & n.s. \\
\hline Schlafzeit (h) & $7,2 \pm 0,6$ & $7,1 \pm 0,6$ & $7,1 \pm 0,5$ & n.s. \\
\hline Aufwacher (n) & $12,5 \pm 7,5$ & $12,8 \pm 9,2$ & $11,1 \pm 7,7$ & n.s. \\
\hline Schlafeffizienz (\%) & $94,9 \pm 3,3$ & $94,6 \pm 3,8$ & $95,6 \pm 3,1$ & n.s. \\
\hline
\end{tabular}

Zur Untersuchung der jeweiligen Korrelationen wurde der Spearman-Korrelationskoeffizient berechnet. Der Zusammenhang zwischen Alter und Geschlecht und der Variabilität des AHI wurde in einem multivariaten Test geprüft. Das Signifikanzniveau beträgt jeweils $p<0,05$. Die Werte sind, falls nicht gesondert angegeben, als Mittelwerte und Standardabweichung angegeben.

\section{Ergebnisse}

Der Apnoe-/Hypopnoeindex betrug in der ersten Messung 9,8 \pm $11,7 / \mathrm{h}$, in der zweiten 10,1 $\pm 9,6 / \mathrm{h}$ und in der dritten Messung $8,5 \pm 9,0 / \mathrm{h}$ (Tab. 1, Abb.1).

Die Ergebnisse des AHI und die Ergebnisse der kardiorespiratorischen Parameter, der Anzahl der Schnarchereignisse und auch der Schlafzeit, der Schlafeffizienz und der Anzahl der Aufwacher erwiesen sich als statistisch nicht signifikant unterschiedlich im Verlauf der drei Messungen. Lediglich bezüglich der Anzahl der Tachy-/Bradykardieereignisse, die in Nacht 2 im Vergleich zu Nacht 1 seltener auftraten, trat ein statistisch signifikanter Unterschied auf.

Intraindividuell ist die Variabilität des AHI jedoch zum Teil stark ausgeprägt (Abb.1). Die individuelle Variabilität bei 10 Patienten betrug bis 5 Ereignisse pro Stunde. Bei 9 Patienten unterschieden sich die Messwerte um mindestens 5 Ereignisse pro Stunde, unter denen wiederum 3 Patienten Differenzen von mehr als $10 \mathrm{Er}$ eignissen pro Stunde aufwiesen. Somit kam es wiederholt zum Über- bzw. Unterschreiten der Schwellen mit einem AHI $\geq 5, \geq 10$ bzw. $\geq 15 / h$.

Bei Patienten mit höherem AHI besteht eine Tendenz zu größerer Variabilität des AHI (Abb. 2). Beim Vergleich zeigte sich in der Gruppe mit einem $A H I \geq 10 / h$ eine größere AHI-Differenz und entsprechend eine größere intraindividuelle Standardabweichung als in der Gruppe mit einem AHI unter 10/h (Tab. 2).

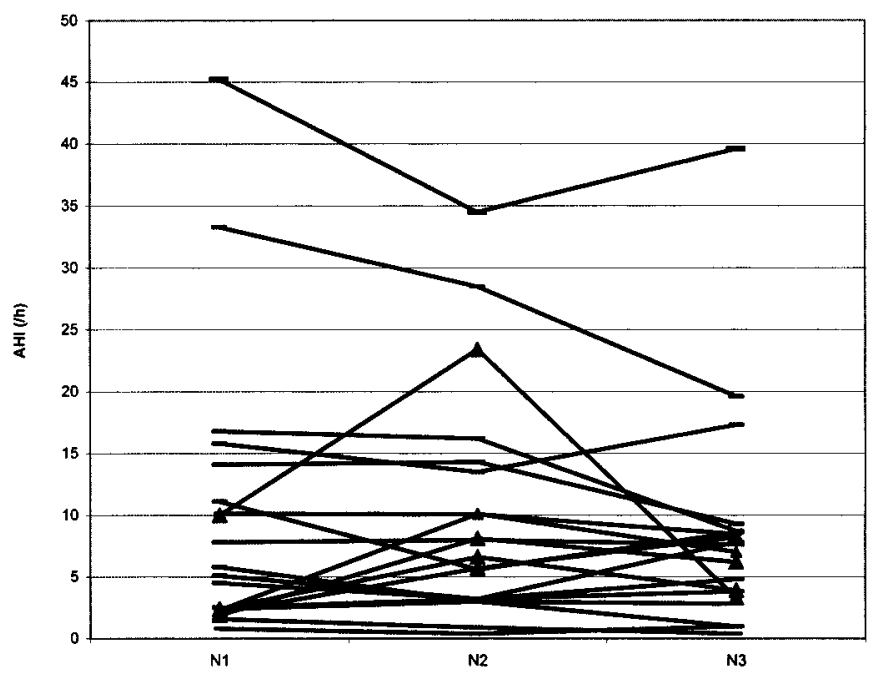

Abb. 1 Individuelle Variabilität des AHI in den 3 Messnächten.

9 Patienten erfüllten das Kriterium eines lageabhängigen AHI. Die Gesamtzahl der nächtlich aufgetretenen Apnoen und Hypopnoen wurde zum Anteil der in Rückenlage aufgetretenen respiratorischen Ereignisse ins Verhältnis gesetzt. Die AHI-Differenz war mit 6,6 $\pm 6,5 / \mathrm{h}$ nicht signifikant unterschiedlich zu den Patienten ohne lageabhängigen AHI-Differenz mit 5,9 4,3/h.

Auch in den 2 Altersgruppen mit einem Alter $<50$ bzw. $\geq 50$ Jahren war kein Unterschied bezüglich der Variabilität des AHI in 3 Nächten nachweisbar, ebenso fanden sich keine geschlechtsspezifische Unterschiede.

Unter der Annahme, dass bei allen Patienten ein Schlafapnoesyndrom vorliegt, liegt bei einer diagnostischen Grenze von $\mathrm{AHI} \geq 5 / \mathrm{h}$ die Sensitivität der zweiten nächtlichen Messung mit 63,2\% über der der ersten Messung mit 52,6\%. Bei Anwendung einer höheren diagnostischen Schwelle von $A H I \geq 10 / h$ und $\mathrm{AHI} \geq 15 / \mathrm{h}$ sind in der ersten und zweiten nächtlichen Messung mit 36,8 bzw. 21,1\% identisch große Sensitivitäten zu beobach- 


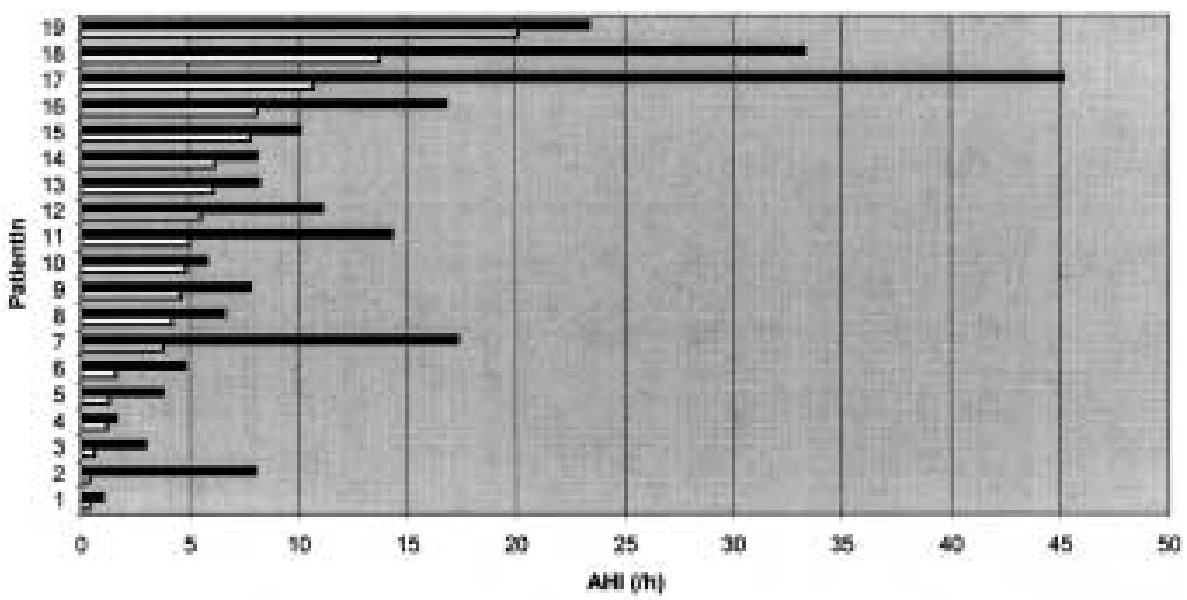

Abb. 2 Darstellung der AHI-Differenz (hell) jedes Patienten und des entsprechenden maximalen $\mathrm{AHI}$ (schwarz).

Tab. 2 Vergleich der AHI-Differenz in der Gruppe mit einem max. $\mathrm{AHI}<10 / \mathrm{h}$ bzw. $>10 / \mathrm{h}$

\begin{tabular}{|c|c|c|c|}
\hline & $\begin{array}{l}\text { Gruppe } 1 \\
\max . A H I<10 / h \\
(n=11)\end{array}$ & $\begin{array}{l}\text { Gruppe } 2 \\
\max . A H I \geq 10 / h \\
(n=8)\end{array}$ & $\begin{array}{l}p \\
\text { T-Test }\end{array}$ \\
\hline $\max . A H I(/ h)$ & $5,3 \pm 2,7$ & $21,4 \pm 12,2$ & $<0,001$ \\
\hline AHI-Differenz (/h) & $2,9 \pm 2,3$ & $9,3 \pm 5,4$ & $<0,002$ \\
\hline
\end{tabular}

Tab. 3 Sensitivität der einzelnen nächtlichen Messungen bei verschiedenen cut-off points des $\mathrm{AHI}$

\begin{tabular}{lllllll}
\hline & N1 & & N2 & & N3 & \\
$\boldsymbol{A H I}(/ \mathbf{h})$ & $\boldsymbol{n}$ & \% & $\boldsymbol{n}$ & $\%$ & $\boldsymbol{n}$ & $\%$ \\
\hline$\geq 5$ & 10 & 52,6 & 12 & 63,2 & 11 & 57,9 \\
$\geq 10$ & 7 & 36,8 & 7 & 36,8 & 3 & 15,8 \\
$\geq 15$ & 4 & 21,1 & 4 & 21,1 & 3 & 15,8 \\
\hline
\end{tabular}

ten. In der 3. Messung liegt die Sensitivität mit 15,8\% jeweils darunter (Tab. 3).

Wird zu der ersten Messung die zweite Messung hinzugenommen, wird die Sensitivität in Bezug auf die cut-off points von $\mathrm{AHI} \geq 5 / \mathrm{h}, \geq 10 / \mathrm{h}, \geq 15 / \mathrm{h}$ um $40,14,3$ bzw. 31,6\% gesteigert und betrug für beide Messungen gemeinsam 73,7\%, 42,1\% bzw. 26,3\%. Auch wenn zur Messung 1 die 3. Messung hinzugezogen wurde, kam es zu einer Sensitivitätssteigerung um 30\% bei einem Cutoff-Wert $\geq 5 / \mathrm{h}$, während es bei einer Betrachtung von Nacht 2 und 3 nur einer Zunahme von 8,3\% im Vergleich zu Einzelmessungen kam. Eine weitere Zunahme der Sensitivität durch gemeinsame Betrachtung der Ergebnisse aus allen drei Messungen war nicht nachweisbar.

\section{Diskussion}

Die Ergebnisse ambulanter polygraphischer Messungen erwiesen sich in verschiedenen Untersuchungen als zuverlässig in der Früherkennung und Therapiekontrolle des Schlafapnoesyndroms [12-14]. Dieses fand Eingang in ein Stufenkonzept zur Diagnostik, wie es von der Deutschen Gesellschaft für Pneumologie [15] empfohlen wird.

Bei ambulanten nächtlichen Aufzeichnungen reichte die Speicherkapazität bislang lediglich für die Aufzeichnung einer Nacht. In der vorliegenden Studie konnte bedingt durch Erhöhung des Speicherplatzes die Messdauer auf 3 Nächte erweitert werden, so dass erstmals prospektiv drei konsekutive ambulante Messungen erfolgten, bei denen die Patienten selbständig Messungen über drei Nächte vornahmen.

Die Korrelation jeweils zweier Nächte sprechen mit Werten zwischen 0,71 und 0,76 für die gute Reproduzierbarkeit der Daten der Gesamtgruppe. Demgegenüber weist ein durchschnittlicher Variationskoeffizient von 30\% auf die weite Streuung der 3 individuellen Messwerte um den Mittelwert hin. Die Indizes variieren bei 3 Patienten um mehr als 10 Ereignisse pro Stunde, bei 6 Patienten zwischen 5 und 10 und bei 10 Patienten um weniger als 5 Ereignisse pro Stunde.

Im Gegensatz zu überwachten Messungen im Schlaflabor war selbst bei genauer Instruktion der Patienten eine hohe Fehleranfälligkeit zu verzeichnen, so dass lediglich in 60 Prozent der Fälle eine Aufzeichnung über 3 Nächte gelang. Insgesamt wurde bei allen Patienten mindestens eine komplette Messung gewonnen, so dass als Ursache nicht eine mangelnde Instruktion wesentlich verantwortlich ist, sondern Fehler bzw. Ausfälle beim wiederholten Anlegen der Sensoren.

Andere Autoren [15-18] fanden bei wiederholten polysomnographischen Messungen eine AHI-Differenz von mehr als 10/h bei $18 \%$ bis zu 50 . Mit $15,8 \%$ ist der Anteil von Patienten mit hochvariablen Befunden hier niedriger. In der vorliegenden Studie wurden Patienten mit klinischem Verdacht auf ein Schlafapnoesyndrom eingeschlossen und somit hatten einige Patienten auch einen unauffälligen AHI, während in den anderen Studien 
meist Patienten mit einem pathologischen Ausgangsbefund in die Variabilitätsmessung aufgenommen wurden $[1-5,16,17,20]$.

In mehreren Studien konnte eine Assoziation zwischen dem Schweregrad des AHI und dem Ausmaß der Variabilität des AHI gezeigt werden [5,17-19]. In der vorliegenden Studie in der Patientengruppe mit einem maximalen $A H I \geq 10 / h$ war eine statistisch signifikant größere AHI-Differenz und Standardabweichung vom Mittelwert des individuellen AHI nachzuweisen als in der Gruppe mit einem geringeren AHI. Bei vergleichbarer Höhe des Variationskoeffizienten unterscheidet sich das Ausmaß der Streuung der individuellen Messwerte um den individuellen Mittelwert in beiden Gruppen nicht. Relativ gesehen weist die Spannbreite der gemessenen Werte in beiden Gruppen eine vergleichbare Größe auf. Mosko u. Mitarb. [19] fanden, dass bei gegebenem AHI mit zunehmender Höhe immer größere Schwankungen des AHI in weiteren Messungen zu erwarten sind, die so ausgeprägt sein können, dass sogar Patienten mit schwergradigen Befunden in einer Screeninguntersuchung oder einmaligen polysomnographischen Messung einen Normalbefund aufweisen können. Zwar scheint mit höhergradigen Befunden eine statistisch signifikante Zunahme der Variabilität einherzugehen, jedoch wurde in allen Studien auch Fälle beobachtet, in denen Patienten mit niedrigem $\mathrm{AHI}$ eine vergleichsweise hohe Variabilität und Patienten mit hohem AHI eine niedrige Variabilität aufwiesen $[2-4,10,11,20]$.

Die natürliche Schlafsituation des Patienten kann durch das Tragen der Messsensoren und im Fall einer stationär durchgeführten polysomnographischen Untersuchung zusätzlich durch eine fremde Umgebung verändert werden. In den Messergebnissen werden diese Veränderungen durch eine verminderte Schlafzeit, einen verringerten Anteil tieferer Schlafstadien und speziell des REM-Anteils sichtbar, die mit dem Begriff First Night Effect (FNE) umschrieben werden [6]. Es ist vorstellbar, dass somit respiratorische Störungen in der ersten Nacht unterschätzt werden. Bei einem Großteil der Studien, in denen polysomnographische Daten ausgewertet wurden, konnte ein klassischer FNE nachgewiesen werden $[2,3,5,16,19]$. Demgegenüber ergab der Vergleich von Ergebnissen wiederholter ambulanter Messungen keinen Hinweis auf einen FNE [7,8,20]. Mit diesen Daten übereinstimmend fand sich auch in der vorliegenden ambulanten Untersuchung kein statistisch nachweisbarer FNE, d.h. im Gegensatz zu einer stationären Polysomnographie ist bei einer ambulanten Messung kein FNE zu erwarten.

Bei einem Teil der Patienten mit obstruktivem Schlafapnoesyndrom wird eine variable Ausprägung des AHI in Abhängigkeit von der Körperlage beobachtet. In einer Studie von Oksenberg u. Mitarb. [9], in der 574 Polysomnogramme unter dieser Fragestellung retrospektiv ausgewertet wurden, fand sich ein Anteil von $55,9 \%$ mit lageabhängigem AHI. In der vorliegenden Studie wurde ein Anteil von etwa $50 \%$ an Patienten mit lageabhängigem AHI gefunden. Es zeigte sich jedoch, dass individuell auch der AHI in Rückenlage in den 3 Messungen variierte. Die AHI-Differenz war bei den Patienten mit lageabhängigem AHI mit 6,6 \pm 6,6/h im Vergleich zu einer AHI-Differenz von 5,9 \pm 4,3/h bei Patienten ohne lageabhängigem AHI statistisch nicht signifikant unterschiedlich ausgeprägt. Übereinstimmend war in allen bis- her erfolgten Untersuchungen eine Lageabhängigkeit der Variabilität des AHI nicht abzuleiten [2,4,5,11,17].

Wie in der vorliegenden Untersuchung konnte auch von Le Bon u. Mitarb. ein Einfluss des Geschlechts der Patienten auf das Ausmaß der individuellen Variabilität des Apnoe-Hypopnoe-Index nicht nachgewiesen werden [5]. Auch das Alter spielte keine Rolle bei der unterschiedlichen Ausprägung des AHI in verschiedenen Nächten $[3,11,18,20]$. Hein u. Mitarb. [11] verglichen den BMI von Patienten mit hochvariablen und niedrigvariablen Befunden. Dabei war die Höhe des BMI nicht mit dem Ausmaß der Variabilität des AHI assoziiert. Gleichzeitig war der zuvor polysomnographisch ermittelte AHI in den untersuchten Gruppen vergleichbar [11].

Entsprechend der Datenlage stellen Alter und Geschlecht keine signifikanten Faktoren bezüglich des Ausmaßes der Variabilität dar, während die Frage bezüglich des BMI nicht eindeutig beantwortet wird.

Alkohol und Sedativa können sich auf einer täglichen Basis begünstigend auf die Ausprägung einer schlafbezogenen Atemstörung auswirken [21]. Hein u. Mitarb. bestimmten anhand morgendlicher Urinkontrollen den Spiegel von Alkohol sowie Sedativa, um Unterschiede im täglichen Konsum als Faktor für die Variabilität auszuschließen. Dabei war keine Abhängigkeit der Variabilität des AHI von den gemessenen Spiegeln nachzuweisen [11]. Ein Einfluss des Wochentags auf die Höhe des AHI ließ sich nicht nachweisen $[3,5]$.

Bei der Wahl einer diagnostischen Grenze von $A H I \geq 10 / h$ betrug der Anteil der falsch negativen Diagnosen in Kollektiven mit Verdacht auf ein Schlafapnoesyndrom 50\% [5,16], 55\% [3] und in der hier vorgestellten Studie zwar deutlich weniger mit $14 \%$, aber insgesamt muss bei einer einmaligen Messung von einem relevanten Anteil an Patienten mit falsch negativen Befunden ausgegangen werden.

Entsprechend den bisherigen Erfahrungen wird für die Therapieindikation der maximale AHI herangezogen $[2,3,16]$. Somit ist bezüglich der Diskrepanz der Ergebnisse mehrerer nächtlicher Messungen weniger die Erfassung falsch positiver Befunde relevant. Vielmehr ist die Unterscheidung zwischen negativen und falsch negativen Befunden im klinischen Alltag bedeutsam. In der vorliegenden Studie wurde bei $40 \%$ der Patienten mit initial negativer Messung in der zweiten Messung ein leichtgradiger Befund erhoben. Bei einem weiteren Patienten wurde erst in der zweiten Messung ein mittelgradiger Befund gefunden.

In Untersuchungen anderer Kollektive betrug die Zunahme der Sensitivität auch bei der Wahl einer höheren diagnostischen Grenze von $\mathrm{AHI} \geq 10 / \mathrm{h} 50 \%$ [2], 55\% [3] und immerhin 15\% bei der Durchführung einer Messwiederholung unter Anwendung eines cut-off points von $\mathrm{AHI} \geq 20 / \mathrm{h}[16]$.

Ein Teil der Patienten weist in der ersten nächtlichen Messung einen AHI auf, der einem mittel- bis schwergradigen Schlafapnoesyndrom entspricht, so dass in diesen Fällen bereits die Indikation zu einer Polysomnographie gestellt werden kann. 
Die vorliegende Studie zeigt, dass bei der Untersuchung von Patienten mit klinischem Verdacht auf ein Schlafapnoesyndrom die Ergebnisse der ersten Messung für ein Kollektiv zwar statistisch ebenso repräsentativ sind wie die der Messwiederholungen, jedoch findet sich bei einem Teil der Patienten eine erhebliche individuelle nächtliche Variabilität der Befunde. Angesichts dieser individuellen Variabilität besteht die Gefahr, mit einer einmaligen Messung den minimalen individuellen Apnoe-/Hypopnoeindex zu erfassen und so die Ausprägung der schlafbezogenen Atmungsstörung zu unterschätzen. In der vorliegenden Studie waren vor allem Patienten mit leichtgradigem Befund vom Problem einer falsch negativen initialen Messung betroffen. Da gerade auch bei Patienten mit schwergradigem Befund häufig eine ausgeprägte Variabilität auftritt, besteht bei einmaliger Messung außerdem die Gefahr, den Schweregrad des Befundes falsch einzuschätzen, und so möglicherweise die Dringlichkeit weiterer Maßnahmen nicht optimal zu beurteilen, so dass insgesamt nach den vorliegenden Daten insbesondere bei Diskrepanz zwischen klinischen Befunden und Screeningbefunden eine Messwiederholung zu empfehlen ist.

\section{Literatur}

${ }^{1}$ Wittig RM, Romaker A, Zorick FJ et al. Night-to night consistency of apneas during sleep. Am Rev Respir Dis 1984; 129: 244-246

2 Dean RJ, Chaudhary BA. Negative polysomnogram in patients with obstructive sleep apnea syndrome. Chest 1992; 101: 105-108

${ }^{3}$ Meyer TJ, Eveloff SE, Kline LR et al. One negative polysomnogram does not exclude obstructive sleep apnea. Chest 1993; 103: 756-776

${ }^{4}$ Chediak AD, Acevedo-Crespo JC, Seiden DJ et al. Sleep disordered breathing. Nightly variability in the indices of sleep-disordered breathing in men being evaluated for impotence with consecutive night polysomnograms. Sleep 1996; 19: 589-592

${ }^{5}$ Le Bon $\mathrm{O}$, Hoffmann G, Tecco J et al. Mild to moderate sleep respiratory events. One night may be not enough. Chest 2000; 118: 353-359

${ }^{6}$ Agnew HW, Webb WB, Williams RL. The first night effect: An EEG study for sleep. Psychophysiology 1966; 2: 263-266
${ }^{7}$ Aber WR, Block AJ, Hellard DW et al. Consistency of respiratory measurements from night to night during the sleep of elderly men. Chest 1989; 96: 747-751

${ }^{8}$ Lord S, Sawyer B, O'Connell D et al. Night-to-night variability of disturbed breathing during sleep in an elderly community sample. Sleep 1991; 14: $252-258$

${ }^{9}$ Oksenberg A, Silverberg DS, Arous E et al. Positional vs nonpositional obstructive sleep apnea patients: anthropomorphic, nocturnal polysomnographic, and multiple sleep latency test data. Chest 1997; 112: 629-639

${ }^{10}$ Bliwise DL, Carey E, Dement WC. Nightly variation in sleep-related respiratory disturbance in older adults. Expir Aging Res 1983; 9: 77-81

${ }^{11}$ Hein H, Behnke G, Jörres RA et al. The therapeutic effect of theophylline in mild obstructive sleep apnea/hypopnea syndrome: Results of repeated measurements with portable recording devices at home. Eur J Med Res 2000; 5: 391 - 399

${ }^{12}$ Penzel T, Amend G, Meinzer K et al. MESAM: a heart rate and snoring recorder for detection of obstructive sleep apnea. Sleep 1990; 13: $175-182$

${ }^{13}$ Roos M, Althaus W, Rhiel C et al. Vergleichender Einsatz von MESAM IV and Polysomnographie bei schlafbezogenen Atmungsstörungen (SBAS). Pneumologie 1993; 47: 112-118

14 Minkley P. Sleep Testing: Center and home-based. Respiratory Care 1998; 43: 296-306

${ }^{15}$ Hein H, Raschke F, Köhler D et al. Leitlinie zur Diagnostik und Therapie schlafbezogener Atmungsstörungen beim Erwachsenen. Pneumologie 2001; 55: 339-342

${ }^{16}$ Mendelson WB. Use of the sleep laboratory in suspected sleep apnea syndrome: Is one night enough? Cleve Clin J Med 1994; 61: 299-303

${ }^{17}$ Bliwise DL, Benkert RE, Ingham RH. Factors associated with nightly variability in sleep-disordered breathing in the elderly. Chest 1991; 100: 973-976

${ }^{18}$ Thoman EB, Acebo C, Lamm S. Sleep in the elderly. Stability and instability of sleep in older persons recorded in the home. Sleep 1993; 16: $578-585$

${ }^{19}$ Mosko SS, Dickel MJ, Ashurst J. Night-to-night variability in sleep apnea and sleep-related periodic leg movements in the elderly. Sleep 1988; $11: 340-348$

${ }^{20}$ Redline S, Tosteson T, Boucher MA et al. Measurement of sleep-related breathing disturbances in epidemiologic studies. Assessment of the validity and reproducibility of a portable monitoring device. Chest 1991; 100: 1281 - 1286

${ }^{21}$ Lugaresi E, Coccagna G, Cirignotta F. Snoring and its clinical implications. In: Guilleminault C, Dement WC (Hrsg.). Sleep apnea syndromes. New York: Alan Liss, 1978: $13-21$ 